\title{
Actualité de la Suisse italienne
}

Nicola Gianinazzi

Nous dispensons régulièrement des consultations individuelles à des confrères psychologues et psychothérapeutes, et à titre occasionnel à des instituts de formation suisses et étrangers tout comme à d'autres institutions privées et publiques. Quant au travail inter-associatif, il porte surtout sur la médiation entre la réalité régionale et nationale, la consultation ainsi que la révision et la traduction de textes d'information ou de nature purement technique.

La collaboration fructueuse avec la Istituto Ricerche di Gruppo (IRG) est entretenue pour l'organisation de la formation continue, dans le but de toujours garantir un vaste choix de cours de qualité. Les cours peuvent être ainsi choisis dans le cadre du cursus de formation post-graduée selon le concept ASP Integral.

Par ailleurs, le soussigné fait partie depuis 2018 de la rédaction de Psychotherapie-Wissenschaft - la Suisse italienne et le panorama italien seront plus présents et visibles dans notre vue thématique. Le travail de contact avec des professeurs universitaires de différentes universités et de facultés tessinoises se poursuit. Cette activité intéressante de relations publiques est utile et nécessaire pour enrichir les offres de formation postgrade et pour promouvoir les projets de recherche, ainsi que les contributions de nos revues associatives. Dans ce dernier domaine, la collaboration rédactionnelle est également approfondie avec la revue Psicoterapia e scienze umane (Psychothérapie et sciences humaines), dont Paolo Migone est co-directeur.

Panorama psychothérapeutique tessinois : lancement d'un projet de recherche sur les couples

Un nouveau projet a été lancé pour approfondir les doutes, les difficultés et les incertitudes des personnes face à un aspect aussi central dans la vie de chacun : la relation de couple. Lobjectif principal consiste à suivre et à accompagner les couples pendant un an de leur vie ensemble pour passer en revue les préoccupations, les doutes, les difficultés et les motivations qui

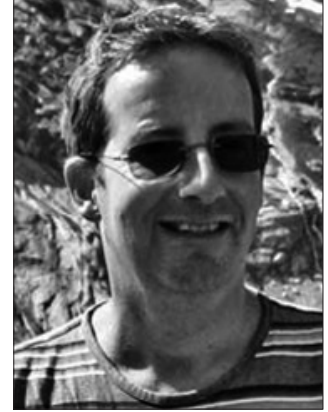

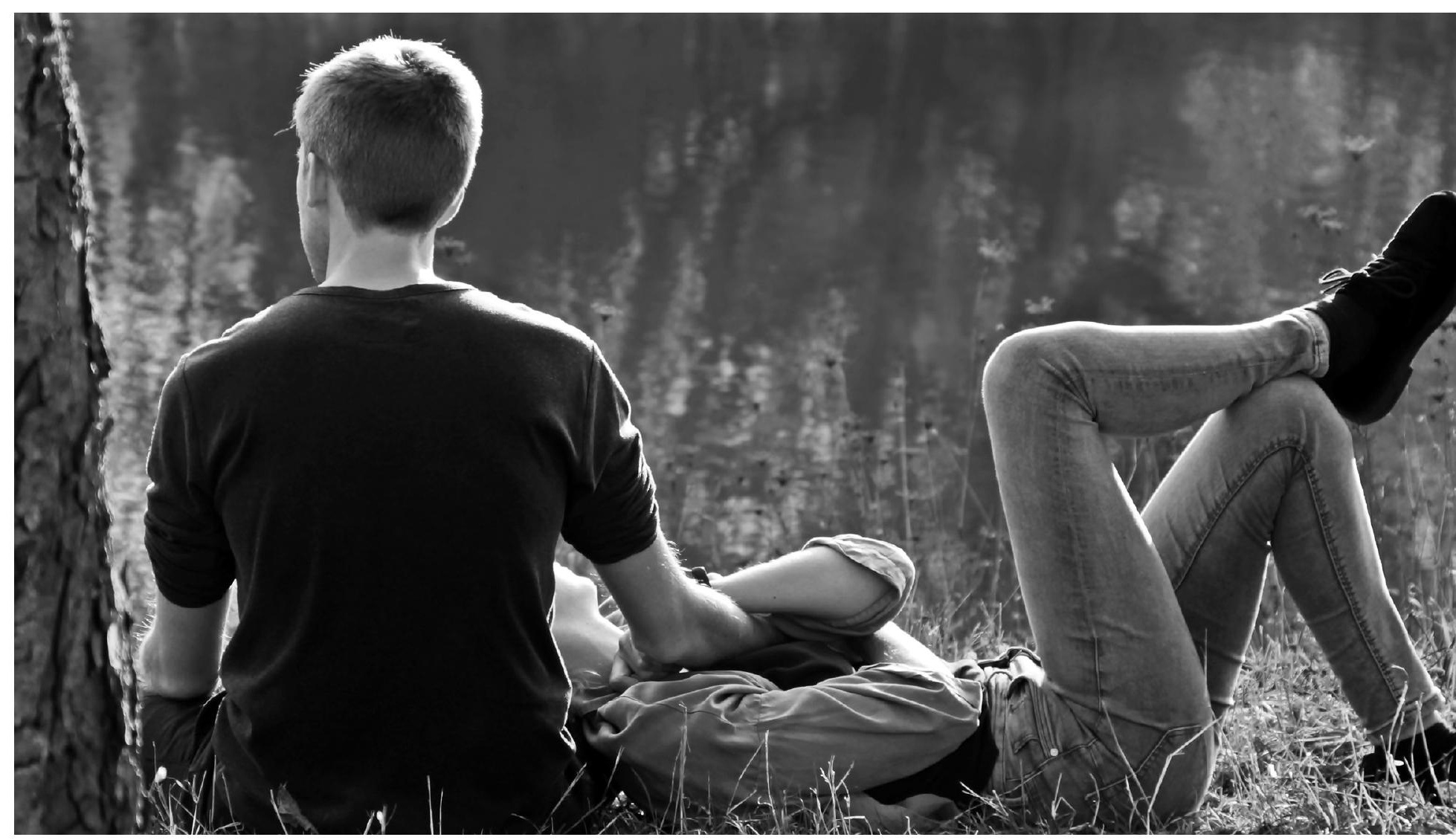


influencent le choix du couple sur la façon de vivre à « long terme » sa propre relation. En effet, une enquête est menée sur certains aspects relatifs au choix de la façon (ou pas) de formaliser sa propre union sentimentale en saisissant l'une des opportunités disponibles sur notre territoire : le mariage civil, le mariage religieux et le concubinage. Si un couple a déjà fait ce choix par le passé, nous lui demandons de nous faire part de leurs préoccupations et des doutes qui ont vécu à cette époque, leur expérience et leur avis comptent pour nous. L'objectif ultime consiste à suivre les couples pendant un an de leur vie ensemble pour évaluer et déceler les ingrédients pour une relation stable et harmonieuse, et les difficultés et les préoccupations que les couples rencontrent quand ils doivent décider s'ils vont vivre leur relation à long terme et de quelle manière.

L'initiative est le fruit d'une collaboration entre des chercheurs de l'IRG (1988) et la Commission diocésaine pour la Pastorale de la famille (1977) : https://www.progettocoppie.ch

Nicola Gianinazzi, membre du comité et délégué pour la Suisse italienne.

\section{Au-delà de la frontière : appel de 600 psychanalystes contre le « Décret de Sécurité »}

« Six-cents psychanalystes de la Società Psicoanalitica Italiana (SPI) ont écrit un appel au Président de la République pour faire entendre leur voix contre le c Décret de Sécurité s et contre les politiques sur la migration du gouvernement jaune et vert. Ils écrivent que la situation, critique depuis longtemps, s'est considérablement envenimée après la promulgation et l'approbation du (Décret de Sécurité >, qui contrairement au terme ‘ sécurité s, est en train de rendre la condition des migrants, et par conséquent celle des italiens, de plus en plus 〈 incertaine ?.

[...] Nous avons conscience que les peurs peuvent aveugler au point de déformer la perception non seulement de
I'autre, mais aussi de sa propre humanité. L'inhumanité est un risque constant où l'être humain peut sombrer sans s'en apercevoir en repoussant toujours plus loin les limites de ce qui est tolérable. C'est pour cela qu'il est d'autant plus nécessaire de savoir écouter ce que la peur occulte pour en faire une possibilité de contact avec soi-même et avec l'autre. Grâce à notre travail de psychanalystes, nous côtoyons des réalités humaines complexes et nous constatons l'urgence de travailler et de réfléchir, même au-delà de notre domaine, sur la possibilité de déceler le ( mal > pour écarter le risque que le < mal > puisse opérer » (https://www.huffingtonpost.it/2019/02/04/seicento-psicoanalisti-si-appellano-a-mattarella-contro-il-decreto-sicurezza_a_23661094). 